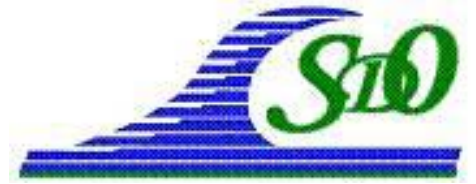

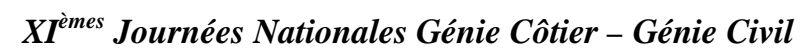

Les Sables d'Olonne, 22-25 juin 2010

DOI:10.5150/jngcgc.2010.055-D @ Editions Paralia CFL

disponible en ligne - http://www.paralia.fr - available online

\title{
Nouvelle méthode pour le suivi environnemental de la zone d'extraction de granulats marins des Graves de Mer
}

\author{
Franck DESMAZES ${ }^{1}$, David CLAVELEAU ${ }^{2}$, \\ Fabrice PLUQUET ${ }^{1}$, Anne-Sophie ALIX ${ }^{1}$
}

1. HOCER, 1 place de Strasbourg, 29200 Brest, France.

franck.desmazes@hocer.com

2. Carrières et Ballastières de Normandie, ZI Zone Bleue,

76370 Rouxmesnil-Bouteilles, France.

david.claveleau@eurovia.com

\section{Résumé :}

Les zones d'extraction de granulats en mer sont soumises à des suivis environnementaux réguliers dans le but de contrôler les quantités prélevées, la position des zones exploitées et l'impact sur l'environnement marin. Pour la zone d'exploitation située au large de Dieppe (Seine-Maritime), l'étude bathymétrique et morphosédimentaire a été confiée au bureau d'étude Hocer par le GIE Graves de Mer. La nouvelle vedette côtière de l'Ifremer, baptisée Haliotis, a été affrétée à cette occasion pour effectuer le levé géophysique au cours du mois de juin 2009. L'utilisation de ce nouvel outil sur une zone d'exploitation en mer constitue l'originalité de ce travail. Cette campagne a permis de réaliser le suivi de l'approfondissement d'un périmètre d'extraction et de tester les outils d'acquisition de la vedette Haliotis dans un environnement sédimentaire extrêmement grossier, constitué majoritairement de galets de silex, et dont le relief est marqué par la présence de cordons de galets et de sillons d'extraction. Après traitement des données, les résultats se sont avérés satisfaisants, permettant de produire un MNT bathymétrique de maille $1 \mathrm{~m}$ et une mosaïque d'imagerie acoustique détaillée, d'une résolution de $40 \mathrm{~cm}$. La morphologie à forts reliefs du site et la présence de sillons générés par l'activité d'extraction par élinde traînante, sont bien reproduites. De plus, l'interprétation des données d'imagerie a permis de mettre en évidence la grande variabilité sédimentaire du secteur.

\section{Mots-clés :}

Extraction de granulats marins - Suivi environnemental - Sondeur interférométrique Bilan sédimentaire

\footnotetext{
Abstract:

According to the French legislation, the marine sand and gravel extraction is subject to regular environmental monitoring to assess the impact on the marine environment. On the extraction site located offshore Dieppe (Seine-Maritime), the bathymetric and sedimentological study has been entrusted to the company Hocer by GIE Graves de
} 
Mer. The new Ifremer vessel, the R/V Haliotis, dedicated to shallow water surveys, has been chartered to perform the geophysical survey in June 2009. Both original objectives were (1) to use this vessel to monitor the dredging activity by assessing the seabed deepening and (2) to test its acquisition tools on a seabed characterized by very coarse sediments (flint pebbles constituting a fossil immerged spit) and a high relief due to extraction furrows made by the drag head. After processing, the data allowed to map a 1-m grid DTM bathymetry and a $40-\mathrm{cm}$ resolution acoustic imagery. The very sensitive response of acquisition tools to the heterogeneous seabed has provided a high definition of the dredging impact through the drag head furrows.

\section{Keywords:}

Marine aggregates extraction - Environmental monitoring - Interferometric sounder Sedimentary assessment

\section{Introduction}

La zone d'extraction de granulats marins des Graves de Mer est située à 8 kilomètres au large de Dieppe (Seine-Maritime), (figure 1). La concession est située sur une flèche littorale fossile, formée essentiellement de galets de silex. Les plages de galets qui forment actuellement le littoral de la région sont des analogues modernes de cette structure sédimentaire sous-marine. Les galets proviennent de l'érosion des falaises côtières lors d'une ou plusieurs périodes de haut niveau marin du Pléistocène (CLAVELEAU, 2007).

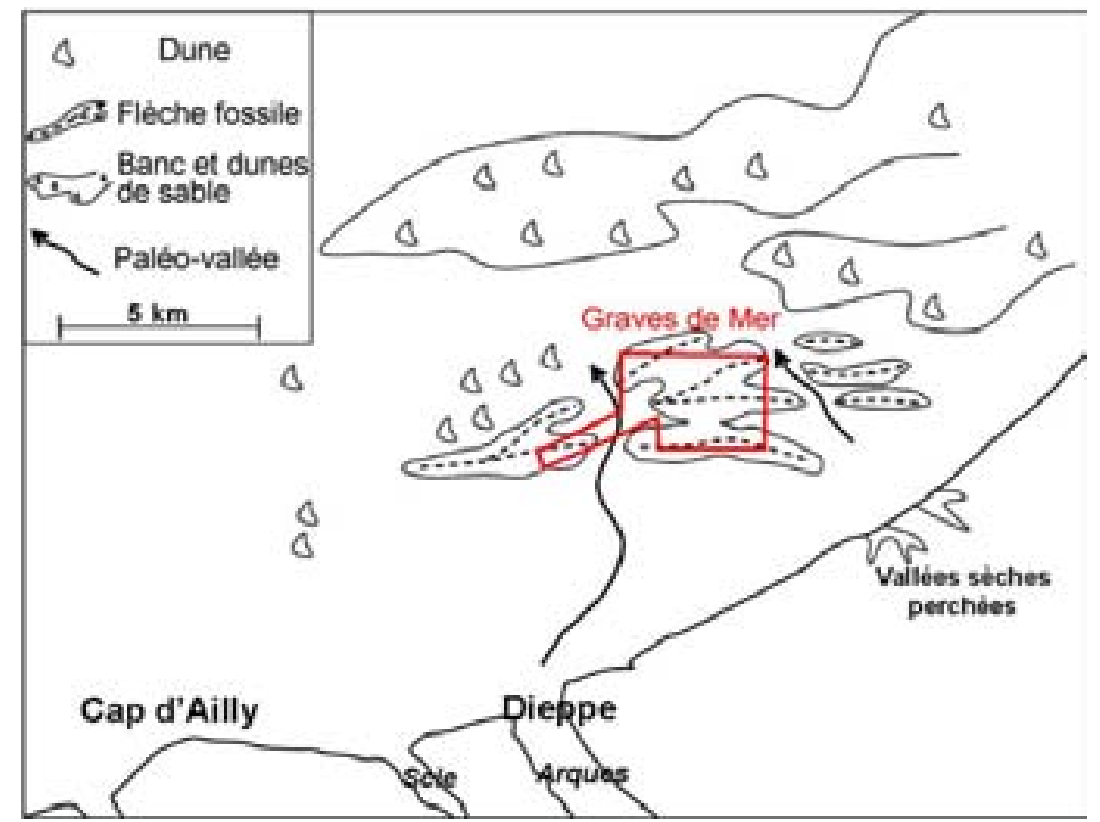

Figure 1. Système de cordons de galets fossiles au large de Dieppe et position de la zone d'extraction des Graves de Mer (CLAVELEAU, 2007). 


\section{XI $I^{\text {èmes }}$ Journées Nationales Génie Côtier - Génie Civil \\ Les Sables d'Olonne, 22-25 juin 2010}

Dans le cadre de son autorisation d'extraction de granulats marins, le GIE Graves de Mer est détenteur d'un permis d'exploitation depuis 1993 sur un périmètre au large de Dieppe (Seine-Maritime). En parallèle, l'entreprise a obtenu, par Arrêté Préfectoral, une autorisation d'Ouverture de Travaux Miniers (OTM) qui fixe les modalités d'exploitation et les mesures de suivi environnemental. Ces dernières visent à déterminer les éventuels effets de l'extraction sur l'environnement tout au long de l'exploitation. Conformément aux préconisations de l'Ifremer, le suivi environnemental est décliné en trois volets :

- le suivi bio-sédimentaire : il est réalisé tous les ans par le biais d'une campagne sédimentaire et benthique qui doit permettre d'apprécier, par rapport à des stations de référence, l'impact sur le benthos des extractions de granulats au droit du secteur autorisé et dans sa périphérie ;

- le suivi bathymétrique : il est destiné à vérifier le respect des profondeurs de dragage et que l'exploitation est correctement effectuée. Un suivi bathymétrique est réalisé tous les 2 millions de tonnes extraites, le délai entre deux mesures ne pouvant pas excéder quatre années ;

- la reconnaissance morpho-sédimentaire : tous les quatre ans, la campagne de levé bathymétrique est complétée par une couverture au sonar à balayage latéral, suivie d'une une récolte d'échantillons pour le calibrage des images acoustiques.

Dans le cadre d'une demande de renouvellement de concession, le GIE Graves de Mer a souhaité mettre en œuvre pour son nouveau suivi morpho-bathymétrique, des méthodes d'acquisition permettant de dresser un état des lieux détaillé avant prolongation de l'activité d'exploitation.

A l'issue d'une phase de consultations, Hocer a proposé une solution innovante basée sur l'utilisation de la nouvelle vedette côtière de l'Ifremer. Les possibilités d'acquisition simultanée des différents paramètres utiles au suivi environnemental et le grand degré de précision du matériel ont conduit le GIE Graves de Mer à adopter cette solution.

\section{Méthode}

\subsection{La vedette Haliotis}

En 2008, l'Ifremer a progressivement fait entrer en opération une nouvelle vedette océanographique, baptisée Haliotis, dotée d'outils acoustiques spécifiques pour l'étude des petits fonds marins de la frange littorale (PLUQUET \& EHRHOLD, 2009). Haliotis est donc une vedette spécialisée, d'une dizaine de mètres de longueur, équipée d'un sonar interférométrique, d'un sondeur à sédiments et d'un sondeur monofaisceau associé à un système de caractérisation des fonds. L'ensemble des équipements électroniques (suivi temps réel, acquisition et archivage des données) est installé à poste fixe dans la cabine. Un pilote mécanicien, un opérateur électronicien et jusqu'à 2 scientifiques peuvent y prendre place. La vedette peut être déplacée entre les sites 
d'étude, par la route, au moyen d'un camion et de sa remorque (figure 2). La mise en œuvre de la vedette est assurée par une grue disposée sur la remorque, afin de permettre la connexion avec l'espace maritime. Cet ensemble permet à Haliotis d'accéder au littoral en complète autonomie.

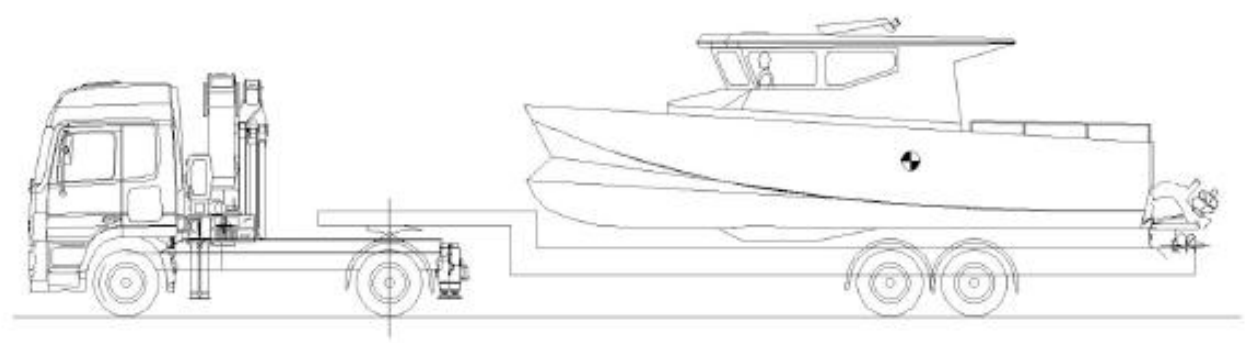

Figure 2. Vedette Haliotis sur son camion de transport. Source : Ifremer.

\subsection{Le sondeur interférométrique Geoswath}

Le sonar interférométrique permet l'acquisition de données de rétrodiffusion, comme un sonar à balayage latéral, sur une largeur de 10 fois la profondeur d'eau. Il permet surtout d'acquérir des données bathymétriques à haute résolution comme un sondeur multifaisceaux, sur une largeur de 5 fois la profondeur d'eau. Par rapport aux méthodes d'acquisition classiques, qui emploient un sondeur multifaisceaux et un sonar latéral à balayage, le système est plus simple à mettre en œuvre et meilleur marché. Dans les zones de forts courants comme la Manche, le positionnement précis de l'imagerie acoustique du sondeur interférométrique prend un avantage décisif par rapport à un sonar latéral remorqué. Le principe de fonctionnement des sonars interférométriques est basé sur l'émission d'une onde acoustique et sur l'enregistrement des retours en fonction de temps (LURTON, 1998). Le sonar qui équipe la vedette Haliotis est un modèle Geoswath Plus de la société GeoAcoustics, il est couplé à une centrale inertielle Hydrins Ixsea qui corrige les mouvements du bateau. Un bathy-célérimètre Valeport est utilisé pour corriger la vitesse du son dans la colonne d'eau.

\subsection{Déroulement de la campagne}

La mission s'est déroulée sur 4 jours, du 22 au 25 juin 2009 dans des conditions météorologiques variables. Au total, 99 kilomètres de profils au sonar interférométrique ont été levés durant la campagne, correspondant à une surface de $8,5 \mathrm{~km}^{2}$. Le système RoxAnn et le sondeur de sédiment ont permis une acquisition en continu. Le positionnement métrique au GPS différentiel qui a été utilisé s'est avéré globalement satisfaisant.

\subsection{Traitement des données}

Le traitement de la bathymétrie et des données d'imagerie acoustique du sonar interférométrique a été réalisé à partir de chaînes de traitement spécifique du logiciel 


\section{XI $I^{\text {èes }}$ Journées Nationales Génie Côtier - Génie Civil \\ Les Sables d'Olonne, 22-25 juin 2010}

Caraïbes 3.4, développé par Ifremer. Le principal travail de post-traitement des données bathymétriques du sondeur Geoswath consiste à épurer les données. Il s'agit d'invalider un maximum de sondes aberrantes. C'est la partie la plus délicate du traitement, car le sondeur interférométrique acquiert de très grandes quantités de sondes. La correction de marée est obtenue, a posteriori, à partir des hauteurs d'eau enregistrées par le marégraphe numérique de Dieppe (données SHOM). La dernière étape de traitement bathymétrique consiste à mailler les données pour réaliser un MNT.

Pour créer une mosaïque d'imagerie acoustique homogène, la navigation et les données de cap ont été filtrées. Les sondes invalidées lors du traitement de bathymétrie n'ont pas été utilisées pour la mosaïque. Le maillage consiste essentiellement à moyenner les données. Une légère interpolation a été réalisée pour améliorer le rendu final. Les différents MNT et images obtenues sont exportés du logiciel Caraïbes afin de permettre le travail d'interprétation sous SIG ArcGis 9.2.

\section{Résultats}

\subsection{Bathymétrie}

Le premier objectif du suivi environnemental est d'observer l'évolution des fonds marins exploités. La figure 3 présente la bathymétrie de la zone d'extraction en juin 2009. La carte montre la présence de trois principaux cordons sédimentaires fossiles au nord-ouest, nord-est et sud de la concession. Les cordons forment des reliefs marqués qui surplombent de 4 à $5 \mathrm{~m}$ de petits talwegs. L'établissement d'un différentiel bathymétrique (figure 4) avec le précédent le levé datant de 2007 montre une érosion nette d'environ $1 \mathrm{~m}$ au niveau du cordon nord-ouest. Dans le reste de la zone, l'érosion est moins flagrante, souvent inférieure à $1 \mathrm{~m}$, sauf au niveau de la bordure nord du cordon sud. Le volume érodé, calculé sur la base de ce différentiel, est en bonne concordance avec les tonnages extraits par le GIE Graves de Mer. L'erreur obtenue est d'environ $10 \%$ et apparaît tout à fait convenable pour ce type d'estimation.

Pour ce travail de suivi bathymétrique, le sondeur interférométrique apparaît comme un outil intéressant, qui permet une couverture globale de la zone, contrairement au précédent levé de 2007 (LEPROUX \& VICAIRE, 2007), réalisé avec un sondeur monofaisceau avec un maillage tous les 50 m, comme le préconise l'Ifremer. En outre, un post-traitement poussé des données bathymétriques et la réalisation de MNT à maille fine (à partir d'un mètre) permettent d'observer les détails du fond marin, comme le relief des sillons d'extraction récents ou l'amplitude des mégarides.

\subsection{Imagerie acoustique}

L'analyse des faciès acoustiques a permis d'identifier (figure 5) :

(1) Des fonds majoritairement constitués de sédiments grossiers (graviers, cailloutis, galets) qui forment les cordons littoraux fossiles. 
Thème 3 - Instrumentation, mesures, imagerie et télédétection

(2) Des fonds de sables moyens, qui présentent des teintes gris moyen à gris clair (faciès peu réflectif) en imagerie acoustique.

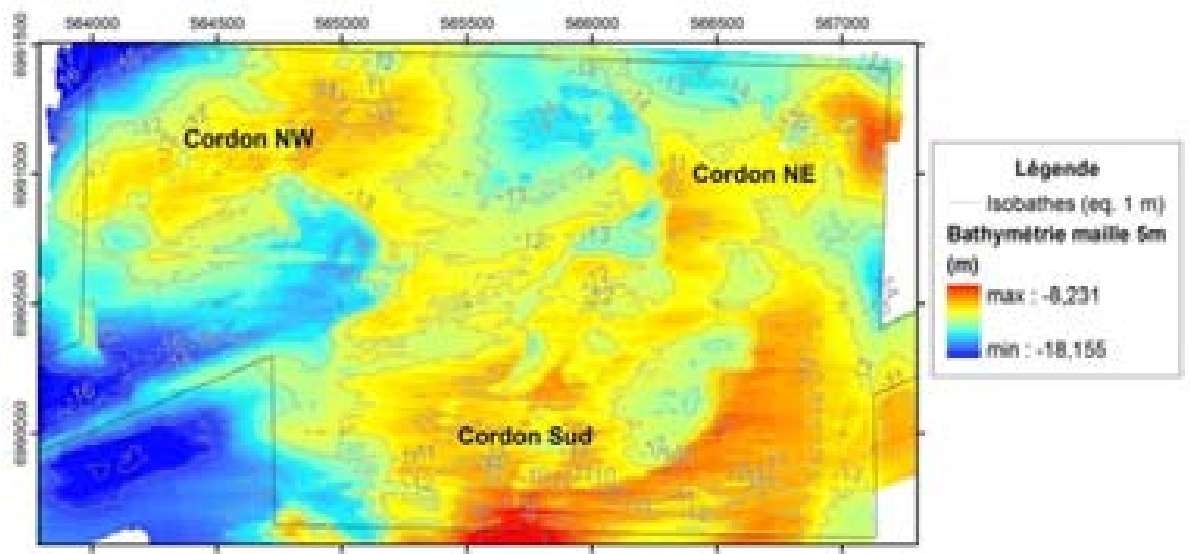

Figure 3. Carte bathymétrique de la zone des Graves de Mer, juin 2009. Maille $5 \mathrm{~m}$. Référentiel: Lambert 93 et zéro hydrographique.

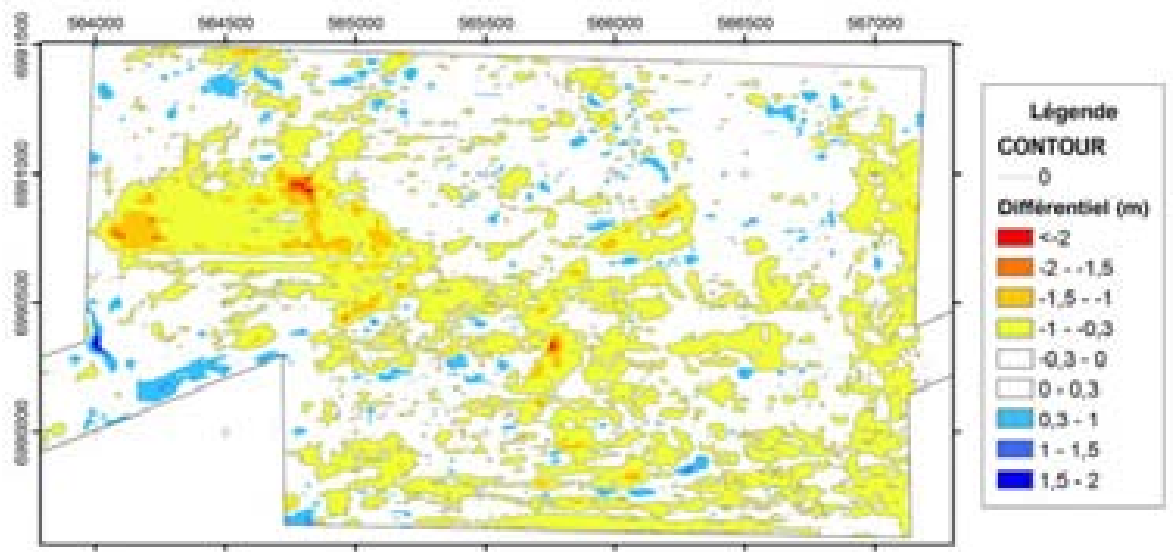

Figure 4. Différentiel bathymétrique entre 2007 et 2009.

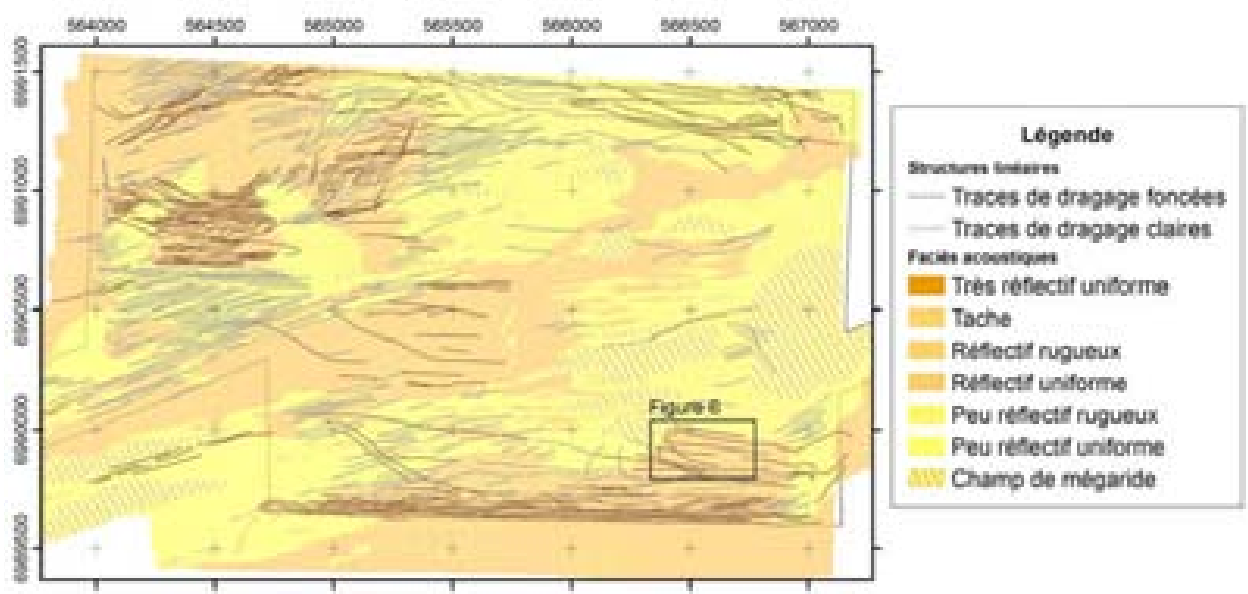

Figure 5. Interprétation de l'imagerie acoustique de la campagne Graves de Mer Haliotis de juin 2009. Référentiel : Lambert 93. 


\section{XI $I^{\text {èes }}$ Journées Nationales Génie Côtier - Génie Civil Les Sables d'Olonne, 22-25 juin 2010}

De très nombreuses traces d'activités humaines sont observées sur la mosaïque d'imagerie acoustique (figure 6). Il s'agit essentiellement des marques laissées par le passage de la tête d'élinde du navire extracteur. Leur densité est particulièrement élevée au NW et en bordure SE de la zone. Par ailleurs, le levé de juin 2009 a été réalisé juste après l'ouverture de la pêche à la seiche dans la bande des 3 milles nautiques. Des traces de panneaux de chalut sont aussi visibles et extrêmement nombreuses sur le fond de la zone d'extraction. Elles se différencient des traces de dragage par une direction très proche de celles des courants de marée. Dans ce domaine complexe, l'origine exacte des différentes traces anthropiques apparait souvent difficile à déterminer car certains sillons d'extraction anciens, remplis par du sable, peuvent se confondre avec des traces de chalutage, voir même avec de possibles rubans sableux.

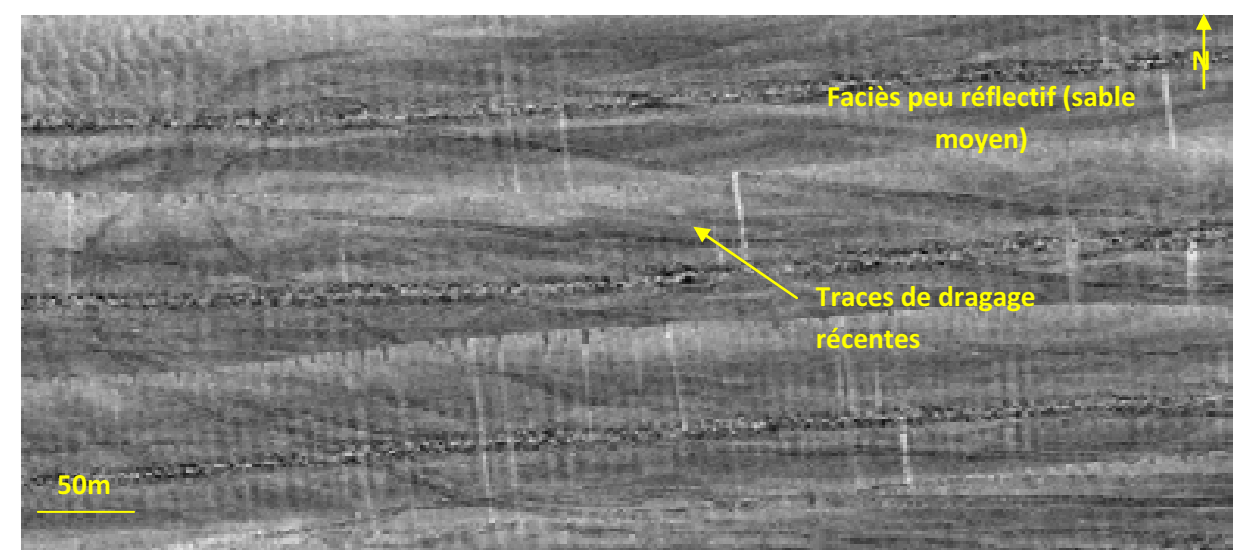

Figure 6. Extrait de mosaïque sonar montrant la présence de traces de dragage récentes (bec d'élinde ou chalutage) sur un fond sableux à grossier.

Enfin, plusieurs champs de mégarides ont été détectés à partir de l'imagerie acoustique (figure 5) et de la bathymétrie. Ces structures sédimentaires remarquables sont visibles au sein de bancs de sables moyens (faciès acoustique peu réflectif). Elles présentent des crêtes rectilignes et une longueur d'onde comprise entre 8 et $10 \mathrm{~m}$. Leur construction résulte d'un transport sédimentaire induit par les courants de marée sur le fond. L'orientation des ces structures indique une direction des courants suivant un axe NESW conformément aux observations antérieures (AUGRIS et al., 1993 ; AUGRIS et al., 2004). En comparaison avec la mosaïque d'imagerie acoustique établie en 2007, il apparaît que la superficie de ces champs de mégarides est en nette croissance. L'augmentation des champs de mégarides est corrélée avec un ensablement visible dans la partie orientale de la zone et déjà signalé en 2007. Une évolution saisonnière de la couverture sableuse est à envisager dans cette zone, mais il est probable que l'ensablement provienne d'une remise en suspension de sable lors du phénomène de surverse inhérent à l'extraction. 


\section{Conclusions}

Dans le cadre du suivi environnemental régulier de la zone d'extraction de granulats marin des Graves de Mer, la société Hocer a affrété l'Haliotis, nouvelle vedette côtière de l'Ifremer. Ce navire, dédié à l'acquisition de données acoustiques par petits fonds apparaît comme un outil particulièrement performant pour le suivi d'une zone d'extraction. La profondeur d'eau moyenne et la faible distance d'un port constituent des conditions idéales pour l'utilisation de la vedette. Il faut cependant noter la sensibilité du navire aux conditions de mer. A partir de 4 Beaufort et dans un clapot en cours de formation, la tenue du navire à la mer et la qualité des mesures peut se dégrader rapidement. En revanche, dans une mer à forte marée, le sondeur interférométrique est un outil puissant car il permet une corrélation et un positionnement parfait de l'imagerie acoustique par rapport à la bathymétrie. Cette caractéristique est particulièrement intéressante pour la cartographie et le repérage précis des sillons d'extraction. Enfin, le sondeur interférométrique est un outil bon marché, puisqu'il remplace avantageusement deux instruments : le sondeur multifaisceau et le sonar latéral à balayage. Pour autant, l'obtention d'une bathymétrie précise et peu bruitée nécessite un effort de post-traitement.

Au final, le sondeur Geoswath a produit de bons résultats dans un domaine constitué de sédiments très grossiers. Le pénétrateur de sédiment de la vedette Haliotis a également fourni des données intéressantes qui peuvent s'avérer précieuses pour estimer les stocks sédimentaires disponibles à l'exploitation.

\section{Références bibliographiques}

AUGRIS C., ClABAUT P., BOURILLET J.F., DREVES L. (1993). Carte morphosédimentaire du domaine marin côtier entre Dieppe et le Tréport (Seine Maritime). Echelle 1/20000. Ifremer édition.

AUGRIS C., ClABAUT P., COSTA S., GOURMELON F., LATTEUX B. (2004) Évolution morpho-sédimentaire du domaine littoral et marin de la Seine-Maritime. Collection Bilans \& prospectives, IFREMER, QUAE, 148 p.

CLAVELEAU D. (2007). Evolution morpho-sédimentaire quaternaire de la plateforme continentale de la Côte d'Albâtre (Manche orientale, France). Thèse Doctorat Univ. Rouen, $243 \mathrm{p}$.

LEPROUX M., VICAIRE O. (2007). Permis des granulats marins de Dieppe. Suivi environnemental et aspects morpho-sédimentaires. Rapport d'étude Astérie et GIE Graves de mer.

LURTON X. (1998). Acoustique sous-marine. Présentation et application. Ifremer édition, $110 \mathrm{p}$.

PLUQUET F., EHRHOLD A. (2009). Une nouvelle stratégie d'étude des habitats marins littoraux au moyen de la vedette acoustique V/O Haliotis. Rapport Ifremer, DYNECO/EB/09-02/FP, 74 p + 4 annexes. 